\title{
Stability Analysis of Two-Segment Stepped Columns with Different End Conditions and Internal Axial Loads
}

\author{
Seval Pinarbasi, Fuad Okay, Erkan Akpinar, and Hakan Erdogan \\ Department of Civil Engineering, Kocaeli University, Umuttepe Campus, 41380 Kocaeli, Turkey \\ Correspondence should be addressed to Seval Pinarbasi; sevalp@gmail.com
}

Received 29 December 2012; Accepted 25 February 2013

Academic Editor: Safa Bozkurt Coskun

Copyright (C) 2013 Seval Pinarbasi et al. This is an open access article distributed under the Creative Commons Attribution License, which permits unrestricted use, distribution, and reproduction in any medium, provided the original work is properly cited.

\begin{abstract}
Members with varying geometrical and/or material properties are commonly used in many engineering applications. Stepped columns with internal axial loads constitute a special case of such nonuniform columns. Crane columns in industrial buildings or structural columns supporting intermediate floors are important applications of stepped members in civil engineering. Since neither axial load nor stiffness is constant along the column height, the stability analysis of a stepped column is usually more complicated than that of a uniform column. Determination of exact buckling loads for stepped columns with different end conditions is not always practical. This paper shows that variational iteration method (VIM), a kind of analytical technique recently proposed for solution of nonlinear differential equations, can satisfactorily be used to obtain approximate solutions for buckling loads of stepped columns with internal axial loads. VIM solutions perfectly match with the exact solutions available in the literature for some special cases of two-segment stepped columns. For many other cases, that is, for various values of three design parameters, namely, (i) load ratio, (ii) stiffness ratio, and (iii) length ratio, approximate buckling loads for two-segment stepped columns are determined using VIM and presented in tabular form which can easily be used by design engineers.
\end{abstract}

\section{Introduction}

Members with functionally graded material distribution and/ or varying cross-sectional dimensions are commonly used in many engineering applications since use of such elements in a structural/mechanical system may reduce the weight of the system considerably, which, in turn, leads to significant cost savings in design. In a geometrically nonuniform member, variation in cross sectional dimensions may be either continuous over the entire length of the member as in tapered members or may occur at discrete points as in stepped members. As an example, an economic design can be achieved in a steel gabled frame if tapered elements are used in the structural system [1]. On the other hand, crane columns commonly used in industrial buildings are frequently designed as stepped columns [2]. Heavy machinery or products moved using cranes, which travel over crane girders, are supported either by column brackets or by stepped columns. Besides the end load coming from the roof system, crane loads produce axial load at an interior point of the column. Stepped columns are also frequently used in multistory structures where columns have to support intermediate floor loads.
Since neither axial load nor stiffness is constant along the column height, the stability analysis of a stepped column is usually more complicated than that of a uniform column. After analyzing each segment of the column separately, the characteristic equation for the entire column has to be obtained using continuity conditions between each segment and boundary conditions at the column ends. In the simplest case, for a two-segment column, such an analysis requires the construction of an eight-by-eight matrix. Finding the smallest root of the resulting characteristic equation can sometimes be rather hard since the results are often very sensitive to the initial guess. Recent studies have already shown that variational iteration method (VIM), a kind of analytical technique proposed by $\mathrm{He}$ [3] for the solution of nonlinear differential equations, can satisfactorily be used to obtain approximate solutions for similar eigenvalue problems. Coşkun and Atay [4], Atay and Coşkun [5], and Okay et al. [6] analyzed the stability of columns with continuously varying stiffness along their lengths using VIM and showed that VIM can effectively be used in stability analysis of nonuniform columns. They also showed that it is much 


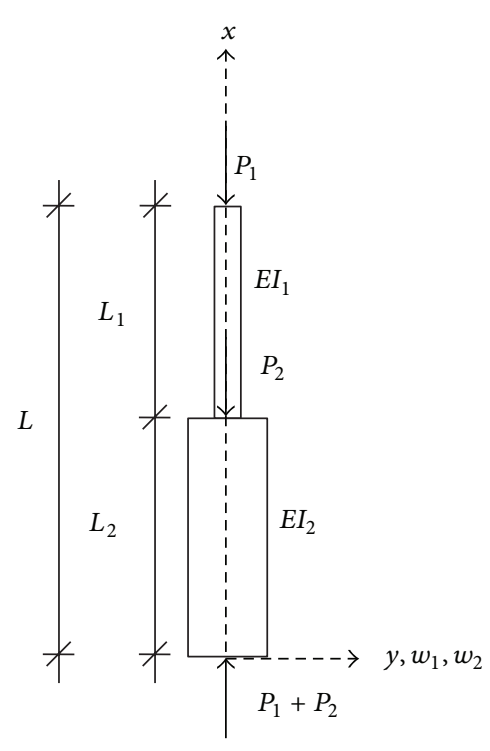

FIGURE 1: An axially loaded stepped column with internal axial load.

easier to determine the buckling loads of uniform columns, for which exact solutions are available in the literature, using VIM since the characteristic equations derived through VIM consist of polynomials. Similarly, Pinarbasi $[7,8]$ has recently applied VIM to lateral torsional buckling analysis of deep rectangular beams whose minor-axis flexural and torsional rigidities change continuously along their lengths and flexural buckling analysis of columns with elastic end restraints whose flexural rigidities vary continuously along their heights and verified that VIM can efficiently be used in these complex stability problems.

In this paper, this practical and powerful technique is applied to stability problems of stepped columns. Although only two-segment stepped columns, which are widely used as crane columns in industrial buildings, are studied, the technique can easily be applied to multisegment stepped columns by following the analysis steps described in the paper to each segment of a multi-segment column. VIM solutions are first compared to the exact solutions available in the literature for some special cases. After verifying the effectiveness of VIM in stability analysis of stepped columns, buckling loads of twosegment stepped columns with different load, stiffness, and length ratios are computed and tabulated using VIM.

\section{Stability Analysis of Stepped Columns with Internal Axial Loads}

Consider a stepped column (Figure 1) compressed by an axial load $P_{1}$ applied at its top end $(x=L)$ besides an axial load $P_{2}$ applied at an intermediate cross-section $\left(x=L_{2}\right)$. Locate the origin of $x-y$ coordinate system at the bottom end of the column. For generality, assume that the upper and lower parts of the column have two different flexural rigidities, $E I_{1}$ for $L_{2} \leq x \leq L_{1}+L_{2}=L$ and $E I_{2}$ for $0 \leq x \leq L_{2}$. Also assume that each end of the column has one of the three classical boundary conditions: clamped (C), pinned (P), or free (F) ends. In order to show the derivation of the buckling equations, let us analyze a column with clamped ends (a C-C column). Figures 2(a) and 2(b) show the undeformed and deformed shapes for such a column. In Figure $2(\mathrm{~b}), M_{A}$, $M_{B}$, and $V$ designate support reactions. Since the flexural rigidities of two segments of the column are different and since there is an internal axial load, we should analyze each segment of the column separately. Equilibrium equation at an arbitrary section in Segment I can be written from the free body diagram shown in Figure 2(c):

$$
E I_{1} \frac{d^{2} w_{1}}{d x^{2}}+P_{1} w_{1}=-V(L-x)+M_{A}
$$

where $w_{1}$ is the displacement of Segment I in $y$ direction. Equation (1) is valid for $L_{2} \leq x \leq L$.

Differentiation of (1) with respect to $x$ gives the shear force in Segment I at any section:

$$
V=E I_{1} \frac{d^{3} w_{1}}{d x^{3}}+P \frac{d w_{1}}{d x}
$$

Further differentiation of (2) with respect to $x$ yields the buckling equation for Segment I:

$$
\frac{d^{4} w_{1}}{d x^{4}}+k_{1}^{2} \frac{d^{2} w_{1}}{d x^{2}}=0, \quad \text { where } k_{1}^{2}=\frac{P_{1}}{E I_{1}} .
$$

Similarly, from Figure 2(d), the equilibrium equation at an arbitrary section in Segment II can be written as

$$
E I_{2} \frac{d^{2} w_{2}}{d x^{2}}+P_{1} w_{2}-P_{2}\left(\delta-w_{2}\right)=-V(L-x)+M_{A},
$$

where $w_{2}$ is the displacement of Segment II in $y$ direction and $\delta$ is the displacement of point $C$, where internal axial load is applied. Equation (4) is valid for $0 \leq x \leq L_{2}$.

Differentiation of (4) with respect to $x$ gives the shear force in Segment II at any section:

$$
V=E I_{2} \frac{d^{3} w_{2}}{d x^{3}}+\left(P_{1}+P_{2}\right) \frac{d w_{2}}{d x}
$$

and further differentiation of (4) gives the buckling equation for Segment II:

$$
\frac{d^{4} w_{2}}{d x^{4}}+k_{2}^{2} \frac{d^{2} w_{2}}{d x^{2}}=0, \quad \text { where } k_{2}^{2}=\frac{\left(P_{1}+P_{2}\right)}{E I_{2}} .
$$

One can easily show that the buckling equations given in (3) and (6) are applicable not only to the columns with clamped ends but also to those with other end conditions.

The solutions of the buckling equations given in (3) and (6), both of which are fourth-order homogeneous differential equations with constant coefficients require eight conditions to determine the resulting eight integration constants. The four of them are written from the continuity conditions where the internal axial load is applied. At this point $(x=$ $L_{2}$ ), the lateral displacement, slope, bending moment, and shear force functions have to be continuous. For the twosegment stepped column shown in Figure 1, these continuity conditions can be written, respectively, as follows:

$$
\left[w_{1}\right]_{x=L_{2}}=\left[w_{2}\right]_{x=L_{2}}, \quad\left[\frac{d w_{1}}{d x}\right]_{x=L_{2}}=\left[\frac{d w_{2}}{d x}\right]_{x=L_{2}},
$$




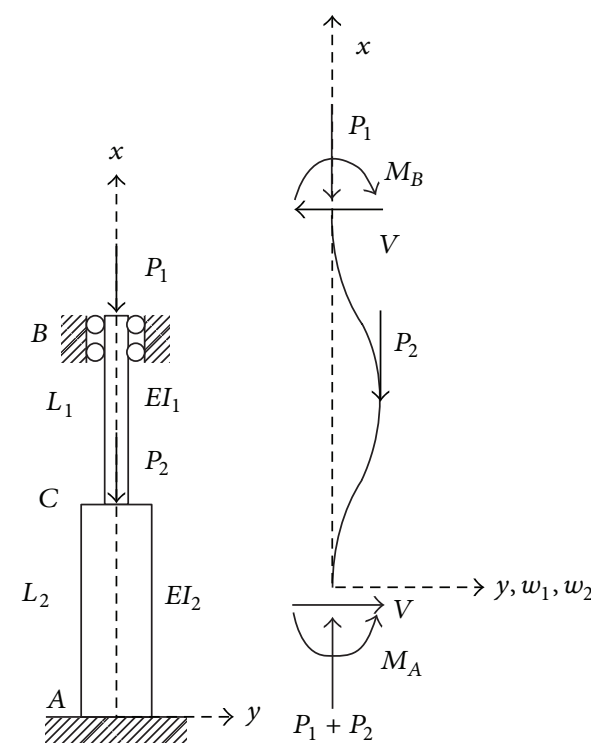

(a) Undeformed shape

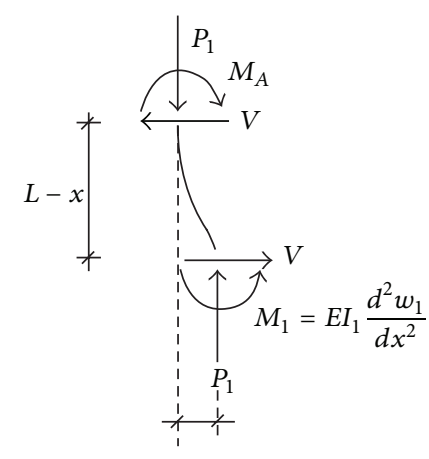

$w_{1}$

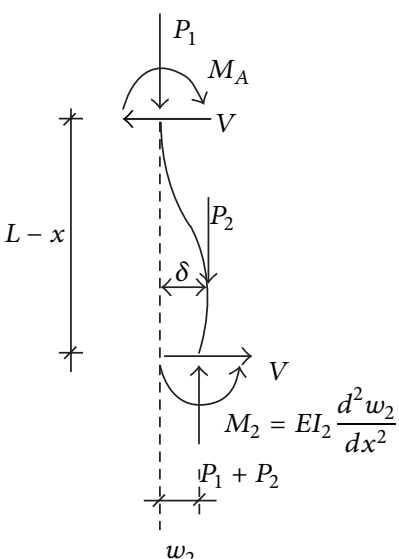

(d) Free body diagram for Segment

FIGURE 2: A fixed (clamped) ended axially loaded stepped column with internal axial load.

$$
\begin{gathered}
{\left[\frac{d^{2} w_{1}}{d x^{2}}\right]_{x=L_{2}}=\frac{E I_{2}}{E I_{1}}\left[\frac{d^{2} w_{2}}{d x^{2}}\right]_{x=L_{2}}} \\
{\left[\frac{d^{3} w_{1}}{d x^{3}}+k_{1}^{2} \frac{d w_{1}}{d x}\right]_{x=L_{2}}=\frac{E I_{2}}{E I_{1}}\left[\frac{d^{3} w_{2}}{d x^{3}}+k_{2}^{2} \frac{d w_{2}}{d x}\right]_{x=L_{2}} .}
\end{gathered}
$$

The other four conditions come from the boundary conditions at the top and bottom ends of the column, which depend on the types of the end supports. For clampedclamped (C-C), clamped-pinned (C-P), clamped-free (C-F), and pinned-pinned (P-P) columns, these conditions can be written as

C-C columns:

$$
\begin{aligned}
& {\left[\frac{d^{2} w_{1}}{d x^{2}}\right]_{x=L}=0,} \\
& {\left[\frac{d^{3} w_{1}}{d x^{3}}+k_{1}^{2} \frac{d w_{1}}{d x}\right]_{x=L}=0,}
\end{aligned}
$$

P-P columns:

$$
\begin{array}{ll}
{\left[w_{2}\right]_{x=0}=0,} & {\left[\frac{d^{2} w_{2}}{d x^{2}}\right]_{x=0}=0,} \\
{\left[w_{1}\right]_{x=L}=0,} & {\left[\frac{d^{2} w_{1}}{d x^{2}}\right]_{x=L}=0 .}
\end{array}
$$

For easier computations, the buckling equations in (3) and (6) can be written in nondimensional form as follows:

$$
\left(\bar{w}_{1}\right)^{\prime \prime \prime \prime}+\beta^{2}\left(\bar{w}_{1}\right)^{\prime \prime}=0, \quad\left(\bar{w}_{2}\right)^{\prime \prime \prime \prime}+\frac{m}{n} \beta^{2}\left(\bar{w}_{2}\right)^{\prime \prime}=0
$$

with

C-F columns:

$$
\left[w_{2}\right]_{x=0}=0, \quad\left[\frac{d w_{2}}{d x}\right]_{x=0}=0,
$$

$$
\beta=k_{1} L, \quad m=\frac{\left(P_{1}+P_{2}\right)}{P_{1}}, \quad n=\frac{E I_{2}}{E I_{1}},
$$


TABLE 1: Comparison of VIM results for $\beta$ values $(s=0.5)$ with exact results (from (23)).

\begin{tabular}{|c|c|c|c|c|c|c|c|c|c|c|}
\hline \multirow{3}{*}{$m$} & \multicolumn{10}{|c|}{$n$} \\
\hline & \multicolumn{2}{|c|}{1} & \multicolumn{2}{|c|}{1.25} & \multicolumn{2}{|c|}{1.5} & \multicolumn{2}{|c|}{1.75} & \multicolumn{2}{|c|}{2} \\
\hline & Exact & VIM & Exact & VIM & Exact & VIM & Exact & VIM & Exact & VIM \\
\hline 1.00 & 3.1416 & 3.1416 & 3.3065 & 3.3065 & 3.4246 & 3.4246 & 3.5124 & 3.5124 & 3.5799 & 3.5799 \\
\hline 1.25 & 2.9608 & 2.9608 & 3.1258 & 3.1258 & 3.2453 & 3.2453 & 3.3348 & 3.3348 & 3.4039 & 3.4039 \\
\hline 1.50 & 2.8065 & 2.8065 & 2.9703 & 2.9703 & 3.0898 & 3.0898 & 3.1800 & 3.1800 & 3.2500 & 3.2500 \\
\hline 1.75 & 2.6732 & 2.6732 & 2.8348 & 2.8348 & 2.9537 & 2.9537 & 3.0439 & 3.0439 & 3.1142 & 3.1142 \\
\hline 2.00 & 2.5566 & 2.5566 & 2.7157 & 2.7157 & 2.8335 & 2.8335 & 2.9232 & 2.9232 & 2.9934 & 2.9934 \\
\hline 3.00 & 2.2050 & 2.2050 & 2.3529 & 2.3529 & 2.4642 & 2.4642 & 2.5502 & 2.5502 & 2.6181 & 2.6181 \\
\hline
\end{tabular}

where $\bar{w}_{1}=w_{1} / L, \bar{w}_{2}=w_{2} / L, \bar{x}=x / L$, and prime denotes differentiation with respect to $\bar{x}$. Similarly, the continuity and boundary conditions can be written in nondimensional form:

$$
\begin{array}{rl}
{\left[\bar{w}_{1}\right]_{\bar{x}=s}} & =\left[\bar{w}_{2}\right]_{\bar{x}=s}, \\
{\left[\left(\bar{w}_{1}\right)^{\prime}\right]_{\bar{x}=s}} & =\left[\left(\bar{w}_{2}\right)^{\prime}\right]_{\bar{x}=s}, \\
{\left[\left(\bar{w}_{1}\right)^{\prime \prime}\right]_{\bar{x}=s}} & =n\left[\left(\bar{w}_{2}\right)^{\prime \prime}\right]_{\bar{x}=s}, \\
{\left[\left(\bar{w}_{1}\right)^{\prime \prime \prime}+\beta^{2}\left(\bar{w}_{1}\right)^{\prime}\right]_{\bar{x}=s}} & n\left[\left(\bar{w}_{2}\right)^{\prime \prime \prime}+\frac{m}{n} \beta^{2}\left(\bar{w}_{2}\right)^{\prime}\right]_{\bar{x}=s}, \\
& \quad \text { where } s=\frac{L_{2}}{L},
\end{array}
$$

C-C columns:

$$
\begin{array}{ll}
{\left[\bar{w}_{2}\right]_{\bar{x}=0}=0,} & {\left[\left(\bar{w}_{2}\right)^{\prime}\right]_{\bar{x}=0}=0,} \\
{\left[\bar{w}_{1}\right]_{\bar{x}=1}=0,} & {\left[\left(\bar{w}_{1}\right)^{\prime}\right]_{\bar{x}=1}=0,} \\
\text { C-P columns: } & \\
{\left[\bar{w}_{2}\right]_{\bar{x}=0}=0,} & {\left[\left(\bar{w}_{2}\right)^{\prime}\right]_{\bar{x}=0}=0,} \\
{\left[\bar{w}_{1}\right]_{\bar{x}=1}=0,} & {\left[\left(\bar{w}_{1}\right)^{\prime \prime}\right]_{\bar{x}=1}=0,}
\end{array}
$$

C-F columns:

$\left[\bar{w}_{2}\right]_{\bar{x}=0}=0, \quad\left[\left(\bar{w}_{2}\right)^{\prime}\right]_{\bar{x}=0}=0$,

$\left[\left(\bar{w}_{1}\right)^{\prime \prime}\right]_{\bar{x}=1}=0$

$\left[\left(\bar{w}_{1}\right)^{\prime \prime \prime}+\beta^{2}\left(\bar{w}_{1}\right)^{\prime}\right]_{\bar{x}=1}=0$

P-P columns:

$$
\begin{array}{ll}
{\left[\bar{w}_{2}\right]_{\bar{x}=0}=0,} & {\left[\left(\bar{w}_{2}\right)^{\prime \prime}\right]_{\bar{x}=0}=0,} \\
{\left[\bar{w}_{1}\right]_{\bar{x}=1}=0,} & {\left[\left(\bar{w}_{1}\right)^{\prime \prime}\right]_{\bar{x}=1}=0 .}
\end{array}
$$

\section{VIM Formulation for the Studied Stability Problem}

According to the variational iteration method (VIM), a general homogeneous nonlinear differential equation can be written in the following form:

$$
L w(x)+N w(x)=0
$$

where $L$ is a linear operator and $N$ is a nonlinear operator, and the "correction functional" is

$$
w_{n+1}(x)=w_{n}(x)+\int_{0}^{x} \lambda(\xi)\left\{L w_{n}(\xi)+N \widetilde{w}_{n}(\xi)\right\} d \xi
$$

In (14), $\lambda(\xi)$ is a general Lagrange multiplier that can be identified optimally via variational theory, $w_{n}$ is the $n$th approximate solution, and $\widetilde{w}_{n}$ denotes a restricted variation; that is, $\delta \widetilde{w}_{n}=0$ [3]. As summarized in He et al. [9], for a fourth-order differential equation such as the equations of the problem considered in this paper $(9), \lambda(\xi)$ equals to

$$
\lambda(\xi)=\frac{(\xi-x)^{3}}{6} .
$$

The original variational iteration algorithm proposed by He [3] has the following iteration formula:

$$
w_{n+1}(x)=w_{n}(x)+\int_{0}^{x} \lambda(\xi)\left\{L w_{n}(\xi)+N w_{n}(\xi)\right\} d \xi
$$

In a recent paper, $\mathrm{He}$ et al. [9] proposed two additional variational iteration algorithms for solving various types of differential equations. These algorithms can be expressed as follows:

$$
\begin{gathered}
w_{n+1}(x)=w_{0}(x)+\int_{0}^{x} \lambda(\xi)\left\{N w_{n}(\xi)\right\} d \xi \\
w_{n+2}(x)=w_{n+1}(x)+\int_{0}^{x} \lambda(\xi)\left\{N w_{n+1}(\xi)-N w_{n}(\xi)\right\} d \xi
\end{gathered}
$$


TABLE 2: Comparison of VIM results for $\beta$ values $(n=1)$ with exact results [11].

\begin{tabular}{|c|c|c|c|c|c|c|c|c|c|c|c|}
\hline \multirow{3}{*}{$\mathrm{BC}$} & \multirow{3}{*}{$m$} & \multicolumn{10}{|c|}{$s$} \\
\hline & & \multicolumn{2}{|c|}{0.1} & \multicolumn{2}{|c|}{0.3} & \multicolumn{2}{|c|}{0.5} & \multicolumn{2}{|c|}{0.7} & \multicolumn{2}{|c|}{0.9} \\
\hline & & Exact & VIM & Exact & VIM & Exact & VIM & Exact & VIM & Exact & VIM \\
\hline \multirow{3}{*}{ C-F } & 4 & 1.56686 & 1.56685 & 1.46680 & 1.46680 & 1.23095 & 1.23096 & 1.01112 & 1.01111 & 0.84904 & 0.84904 \\
\hline & 2 & 1.56950 & 1.56950 & 1.53686 & 1.53686 & 1.43779 & 1.43779 & 1.30081 & 1.30082 & 1.16904 & 1.16904 \\
\hline & 1.33 & 1.57036 & 1.57037 & 1.55961 & 1.55961 & 1.52425 & 1.52425 & 1.46498 & 1.46497 & 1.39505 & 1.39506 \\
\hline \multirow{3}{*}{ P-P } & 4 & 2.42298 & 2.42298 & 2.00448 & 2.00448 & 1.96601 & 1.96601 & 1.89495 & 1.89495 & 1.68817 & 1.68816 \\
\hline & 2 & 2.85769 & 2.85769 & 2.59029 & 2.59029 & 2.55657 & 2.55656 & 2.50864 & 2.50864 & 2.33135 & 2.33135 \\
\hline & 1.33 & 3.04226 & 3.04226 & 2.92536 & 2.92536 & 2.90675 & 2.90676 & 2.88509 & 2.88509 & 2.78735 & 2.78735 \\
\hline \multirow{3}{*}{ C-P } & 4 & 4.39908 & 4.39907 & 3.37502 & 3.37502 & 3.01150 & 3.01149 & 2.94442 & 2.94442 & 2.50798 & 2.50798 \\
\hline & 2 & 4.46510 & 4.46509 & 4.06141 & 4.06141 & 3.80247 & 3.80247 & 3.76424 & 3.76424 & 3.42116 & 3.42116 \\
\hline & 1.33 & 4.48429 & 4.48429 & 4.34651 & 4.34652 & 4.22846 & 4.22847 & 4.21207 & 4.21207 & 4.03808 & 4.03808 \\
\hline \multirow{3}{*}{ P-C } & 4 & 3.15057 & 3.15057 & 2.66544 & 2.66544 & 2.62683 & 2.62682 & 2.40572 & 2.40571 & 2.25642 & 2.25641 \\
\hline & 2 & 3.91057 & 3.91057 & 3.54573 & 3.54573 & 3.51694 & 3.51694 & 3.32992 & 3.32992 & 3.18659 & 3.18659 \\
\hline & 1.33 & 4.28124 & 4.28124 & 4.10249 & 4.10249 & 4.08788 & 4.08789 & 3.98602 & 3.98602 & 3.89716 & 3.89716 \\
\hline \multirow{3}{*}{$\mathrm{C}-\mathrm{C}$} & 4 & 5.97573 & 5.97573 & 4.16581 & 4.16580 & 3.93102 & 3.93102 & 3.54153 & 3.54153 & 3.16821 & 3.16821 \\
\hline & 2 & 6.19875 & 6.19876 & 5.32574 & 5.32574 & 5.11300 & 5.11300 & 4.82979 & 4.82978 & 4.46859 & 4.46859 \\
\hline & 1.33 & 6.25686 & 6.25686 & 5.93175 & 5.93175 & 5.81351 & 5.81351 & 5.67979 & 5.67980 & 5.45754 & 5.45753 \\
\hline
\end{tabular}

Thus, the three VIM iteration algorithms for the buckling equations given in (9) can be written as follows:

$$
\begin{array}{r}
\bar{w}_{i, n+1}(x)=\bar{w}_{i, n}(x) \\
\quad+\int_{0}^{x} \frac{(\xi-x)^{3}}{6}\left\{\bar{w}_{i, n}^{\prime \prime \prime \prime}(\xi)+a \beta^{2} \bar{w}_{i, n}^{\prime \prime}(\xi)\right\} d \xi \\
\bar{w}_{i, n+1}(x)=\bar{w}_{i, 0}(x)+\int_{0}^{x} \frac{(\xi-x)^{3}}{6}\left\{a \beta^{2} \bar{w}_{i, n}^{\prime \prime}(\xi)\right\} d \xi \\
\bar{w}_{i, n+2}(x) \\
=\bar{w}_{i, n+1}(x) \\
+\int_{0}^{x} \frac{(\xi-x)^{3}}{6}\left\{\left(\bar{w}_{i, n+1}^{\prime \prime \prime \prime}(\xi)-\bar{w}_{i, n}^{\prime \prime \prime \prime}(\xi)\right)\right. \\
\left.+a \beta^{2}\left(\bar{w}_{i, n+1}^{\prime \prime}(\xi)-\bar{w}_{i, n}^{\prime \prime}(\xi)\right)\right\} d \xi,
\end{array}
$$

where $i$ is the segment number and can take the values of one or two; $a=1$ when $i=1$, and $a=m / n$ when $i=2$.

In order to determine the most effective VIM algorithm to be used in the current study, for various values of problem variables (i.e., "load ratio" $m$, "stiffness ratio" $n$, and "length ratio" s), VIM results are obtained using all three iteration algorithms. Parallel to the findings of Pinarbasi [7], all iteration algorithms yield exactly the same results for this special buckling problem. For this reason, considering its simplicity, the second iteration algorithms given in (18) are decided to be used throughout the study.
For particular values of $m, n$, and $s$, VIM iterations are started with a third-degree polynomial for displacement function of each segment:

$$
\begin{aligned}
& \bar{w}_{1,0}=C_{1}(\bar{x})^{3}+C_{2}(\bar{x})^{2}+C_{3} \bar{x}+C_{4}, \\
& \bar{w}_{2,0}=C_{5}(\bar{x})^{3}+C_{6}(\bar{x})^{2}+C_{7} \bar{x}+C_{8} .
\end{aligned}
$$

Thus, the problem contains eight unknown coefficients $C_{i}(i=1-8)$ which are to be determined from continuity and end conditions. After conducting seventeen iterations, $\bar{w}_{1,17}$ and $\bar{w}_{2,17}$ are obtained. Substituting these approximate solutions to the continuity equations in (11) and to one set of the boundary conditions listed in (12) depending on the support conditions of the ends of the column, eight homogeneous equations are obtained. These equations can be put into the following matrix form:

$$
[M(\beta)]\{C\}=\{0\},
$$

where $\{C\}=\left\{C_{1} C_{2} C_{3} C_{4} C_{5} C_{6} C_{7} C_{8}\right\}^{T}$. Thus, the problem reduces to an eigenvalue problem. For a nontrivial solution, the determinant of the coefficient matrix has to be zero. The smallest possible real root of the characteristic equation, which is obtained by equating the determinant of the coefficient matrix to zero, gives the square root of the nondimensional buckling load $\left(\beta=k_{1} L=\sqrt{P_{1} L^{2} / E I_{1}}\right)$ in the first buckling mode. 
TABLE 3: VIM results for $\beta$ values for $n=2$ and $n=4$.

\begin{tabular}{|c|c|c|c|c|c|c|c|c|c|c|c|}
\hline \multirow{3}{*}{ BC } & \multirow{3}{*}{$m$} & \multicolumn{5}{|c|}{$n=2$} & \multicolumn{5}{|c|}{$n=4$} \\
\hline & & & & $s$ & & & & & $s$ & & \\
\hline & & 0.1 & 0.3 & 0.5 & 0.7 & 0.9 & 0.1 & 0.3 & 0.5 & 0.7 & 0.9 \\
\hline \multirow{4}{*}{$\mathrm{C}-\mathrm{F}$} & 4 & 1.65206 & 1.78368 & 1.68681 & 1.42537 & 1.20064 & 1.69774 & 2.00174 & 2.21430 & 2.00174 & 1.69774 \\
\hline & 2 & 1.65287 & 1.82208 & 1.91063 & 1.82208 & 1.65287 & 1.69796 & 2.01578 & 2.38550 & 2.52250 & 2.33637 \\
\hline & 1.33 & 1.65314 & 1.83391 & 1.99225 & 2.03862 & 1.97192 & 1.69804 & 2.02012 & 2.43740 & 2.78307 & 2.78585 \\
\hline & 1 & 1.65327 & 1.83963 & 2.03334 & 2.17345 & 2.21961 & 1.69808 & 2.02223 & 2.46192 & 2.93360 & 3.13371 \\
\hline \multirow{4}{*}{ P-P } & 4 & 2.44736 & 2.15245 & 2.35390 & 2.60062 & 2.38651 & 2.45939 & 2.23070 & 2.60160 & 3.40546 & 3.37234 \\
\hline & 2 & 2.87062 & 2.73561 & 2.99339 & 3.37891 & 3.29347 & 2.87693 & 2.80819 & 3.25292 & 4.26056 & 4.64699 \\
\hline & 1.33 & 3.04987 & 3.05477 & 3.35033 & 3.82251 & 3.93371 & 3.05358 & 3.11725 & 3.60214 & 4.69679 & 5.53814 \\
\hline & 1 & 3.14659 & 3.25522 & 3.57986 & 4.10715 & 4.42814 & 3.14903 & 3.30900 & 3.82127 & 4.95922 & 6.21690 \\
\hline \multirow{4}{*}{ C-P } & 4 & 4.679810 & 3.970490 & 3.517460 & 3.913770 & 3.545850 & 4.835630 & 4.766060 & 4.059840 & 4.726560 & 5.011600 \\
\hline & 2 & 4.702090 & 4.618720 & 4.348370 & 4.830420 & 4.830060 & 4.841840 & 5.276390 & 4.943270 & 5.653910 & 6.804160 \\
\hline & 1.33 & 4.708810 & 4.851840 & 4.768080 & 5.289040 & 5.687220 & 4.843790 & 5.422040 & 5.365570 & 6.098110 & 7.965020 \\
\hline & 1 & 4.712050 & 4.964310 & 5.018280 & 5.563920 & 6.309090 & 4.844740 & 5.487200 & 5.607940 & 6.359200 & 8.770790 \\
\hline \multirow{4}{*}{ P-C } & 4 & 3.20780 & 2.95096 & 3.33420 & 3.20236 & 2.99877 & 3.23621 & 3.10773 & 3.90356 & 4.32635 & 3.96682 \\
\hline & 2 & 3.94773 & 3.86346 & 4.37165 & 4.39937 & 4.22783 & 3.96570 & 4.02591 & 4.99428 & 5.87100 & 5.58124 \\
\hline & 1.33 & 4.30427 & 4.41335 & 4.99678 & 5.22272 & 5.16151 & 4.31532 & 4.56360 & 5.61479 & 6.86693 & 6.79931 \\
\hline & 1 & 4.50824 & 4.78483 & 5.41993 & 5.83677 & 5.94039 & 4.51536 & 4.91969 & 6.01986 & 7.55381 & 7.80796 \\
\hline \multirow{4}{*}{$\mathrm{C}-\mathrm{C}$} & 4 & 6.49864 & 4.85117 & 4.71153 & 4.79301 & 4.22351 & 6.76096 & 5.79608 & 5.33991 & 6.52958 & 5.62997 \\
\hline & 2 & 6.57166 & 6.05035 & 5.99937 & 6.44874 & 5.93957 & 6.77901 & 7.01275 & 6.71136 & 8.43046 & 7.89341 \\
\hline & 1.33 & 6.59137 & 6.61050 & 6.72061 & 7.45760 & 7.23127 & 6.78432 & 7.47136 & 7.45027 & 9.36667 & 9.57824 \\
\hline & 1 & 6.60050 & 6.90898 & 7.18459 & 8.11453 & 8.29738 & 6.78686 & 7.67979 & 7.91216 & 9.91097 & 10.95100 \\
\hline
\end{tabular}

\section{Comparison of VIM Results with Exact Results for Some Special Cases Available in the Literature}

Timoshenko and Gere [10] solved this buckling problem for a bar with hinged ends, that is, a P-P column. They derived the following characteristic equation for this particular case:

$$
\frac{k_{4}^{2}}{k_{1}^{2}}-\frac{k_{1}^{2} L+k_{4}^{2} L_{1}}{k_{1} \tan k_{1} L_{1}}=\frac{k_{3}^{2}}{k_{2}^{2}}+\frac{k_{2}^{2} L-k_{3}^{2} L_{2}}{k_{2} \tan k_{2} L_{2}}
$$

where

$$
k_{1}^{2}=\frac{P_{1}}{E I_{1}}, \quad k_{2}^{2}=\frac{P_{1}+P_{2}}{E I_{2}}, \quad k_{3}^{2}=\frac{P_{2}}{E I_{2}}, \quad k_{4}^{2}=\frac{P_{2}}{E I_{1}} .
$$

In terms of $m, n, s$, and $\beta$, (21) can be reformulated as follows:

$$
\begin{aligned}
(m-1) & -\frac{\beta[1+(m-1)(1-s)]}{\tan [\beta(1-s)]} \\
& =\frac{(m-1)}{m}+\sqrt{\frac{m}{n}} \frac{\beta[1-s(m-1) / m]}{\tan [\sqrt{m / n} \beta s]} .
\end{aligned}
$$

Exact values computed from (23) are compared with the approximate VIM results in Table 1, which shows perfect match.
Exact solutions are also available for uniform columns. Assuming that the flexural stiffness of the column remains constant along its entire length (i.e., assuming $n=1$ ), Wang et al. [11] derived the characteristic equations for the studied buckling problem and obtained exact results for various end conditions and different values of $m$ and $s$. For the same boundary conditions and $m, s$ values, normalized buckling loads $(\beta)$ are also computed using VIM. VIM results, as well as the exact results, are presented in Table 2.

Comparison of the results presented in Table 2 indicates good agreement between VIM results and the exact results.

\section{VIM Solutions for Various Load, Stiffness, and Length Ratios}

After verifying the effectiveness of VIM in stability analysis of stepped columns, buckling loads of two-segment stepped columns with different load $(m)$, stiffness $(n)$, and length $(s)$ ratios are computed using VIM, and the results are tabulated in Table 3. These results can directly be used by design engineers who design two-segment stepped columns. For C$\mathrm{F}$ and C-C columns, VIM solutions are also plotted in Figures 3 and 4 in a comparative way. It is worth noting that among the columns with the studied five sets of end conditions, C-F columns are those that have the smallest buckling loads, and $\mathrm{C}-\mathrm{C}$ columns are those with the largest capacities. Figures 3(a) and 4(a) clearly show that buckling load of a uniform column, that is, a column with $n=1$, decreases as the internal 


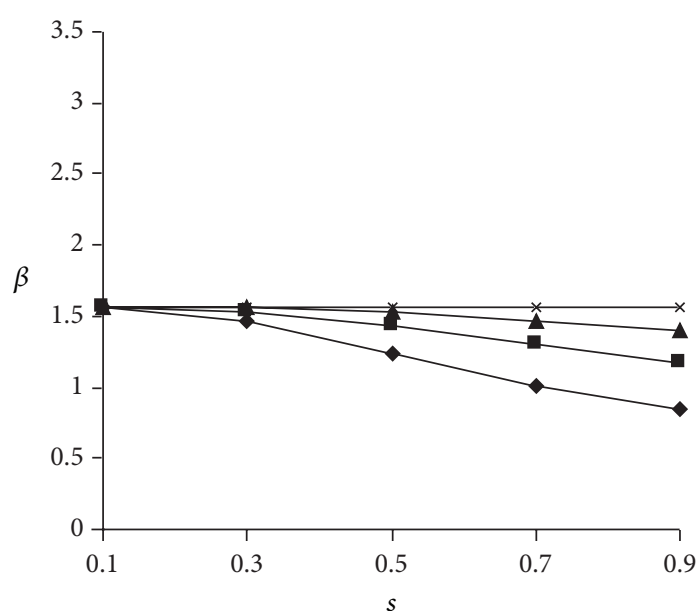

(a) $n=1$

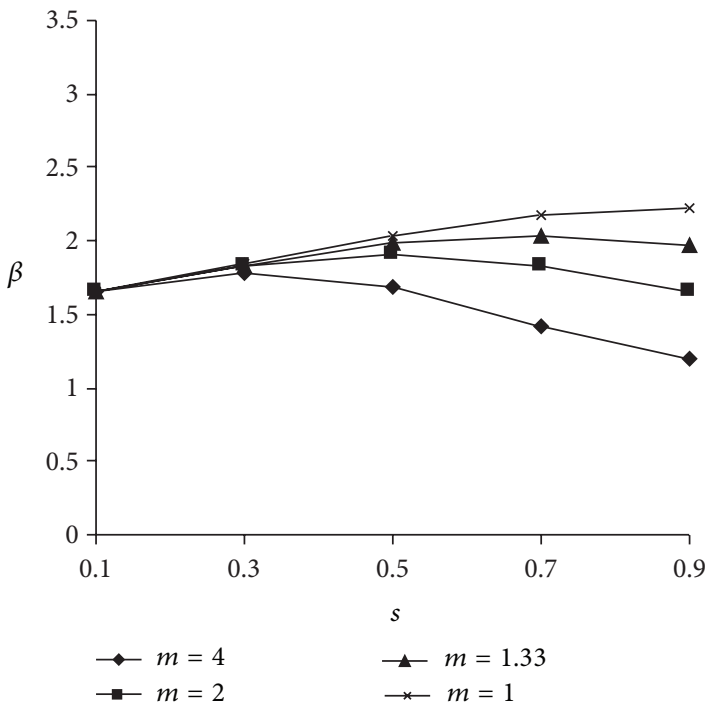

(c) $n=2$

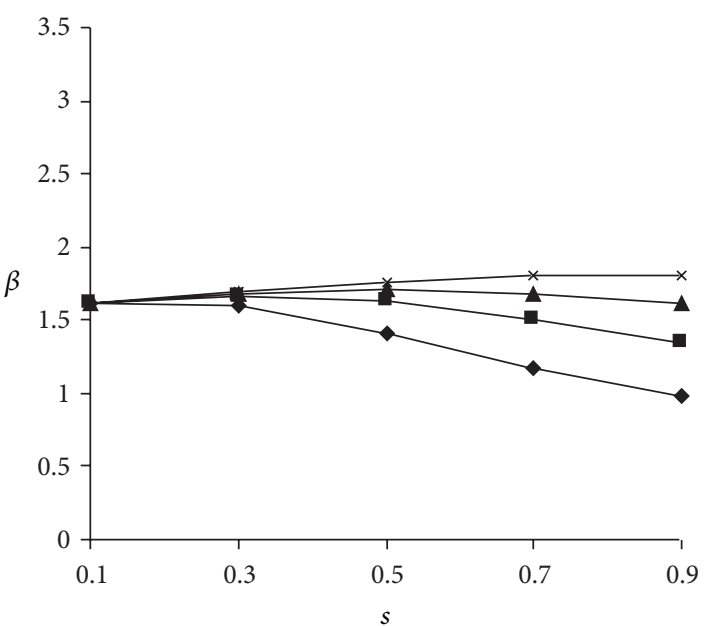

(b) $n=1.33$

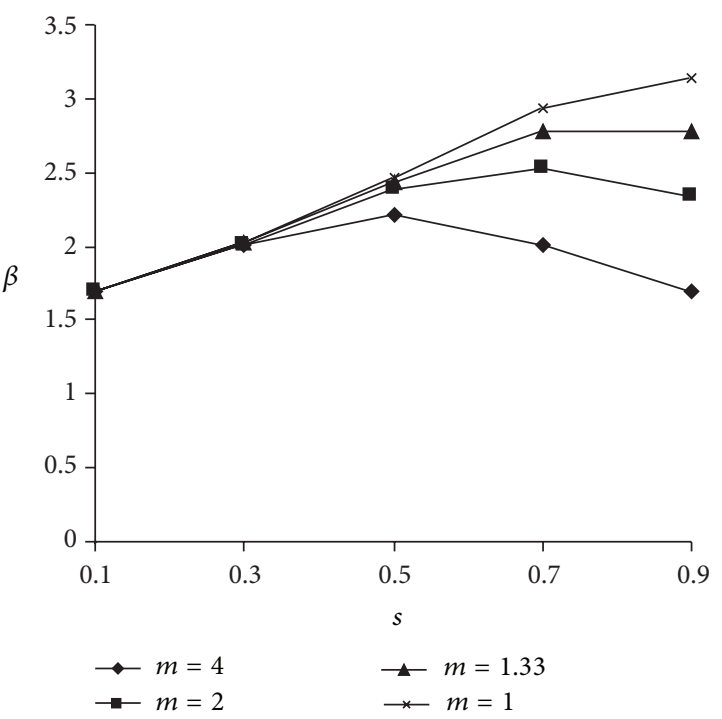

(d) $n=4$

FiguRE 3: VIM results for $\beta$ values for two-segment stepped columns with clamped-free (C-F) ends for different values of load ratio $(m)$, stiffness ratio $(n)$, and length ratio $(s)$.

axial load increases, that is, as $m$ increases, and/or as the application point of the internal axial load moves towards the top end of the column, that is, as $s$ increases. However, as it can be seen from Figures 3(b)-3(d) and Figures 4(b)-4(d), by increasing the stiffness of the lower segment of the column, that is, by increasing $n$, the load that can be carried by the column prior to buckling may be increased significantly. In other words, buckling load of a column can be increased by designing it as a nonuniform column. The effect of $n$ on $\beta$ is more apparent when $s$ is close to unity.

\section{Conclusions}

Structural and mechanical members have traditionally been designed to have constant stiffness along their lengths. The design of such members is usually straightforward since there are thoroughly studied and well-developed guidelines in design specifications for such uniform members. In an attempt to achieve economic designs, more and more engineers recently design nonuniform members with functionally graded material distribution and/or varying cross-sectional dimensions. However, there are very few studies in the literature on behavior and failure mechanisms of nonuniform members under different loading conditions since the analysis of nonuniform members requires the solution of more complex problems. It is usually very hard and/or impractical to obtain exact solutions to these problems due to the complex differential equations derived in the analyses. Recently developed analytical techniques for solution of nonlinear differential equations, such as variational iteration method (VIM), however, can satisfactorily be used to solve such complex problems.

In this study, buckling analysis of two-segment stepped columns with different flexural stiffness at each segment are 


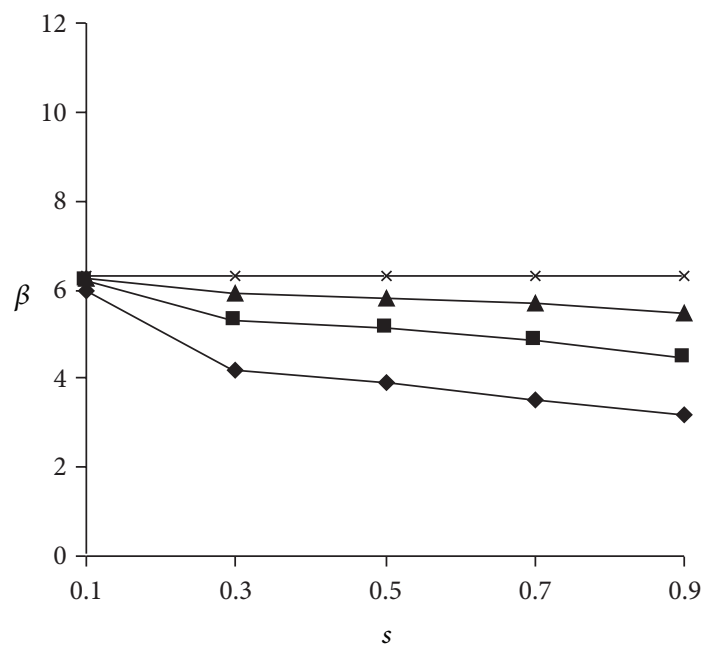

(a) $n=1$

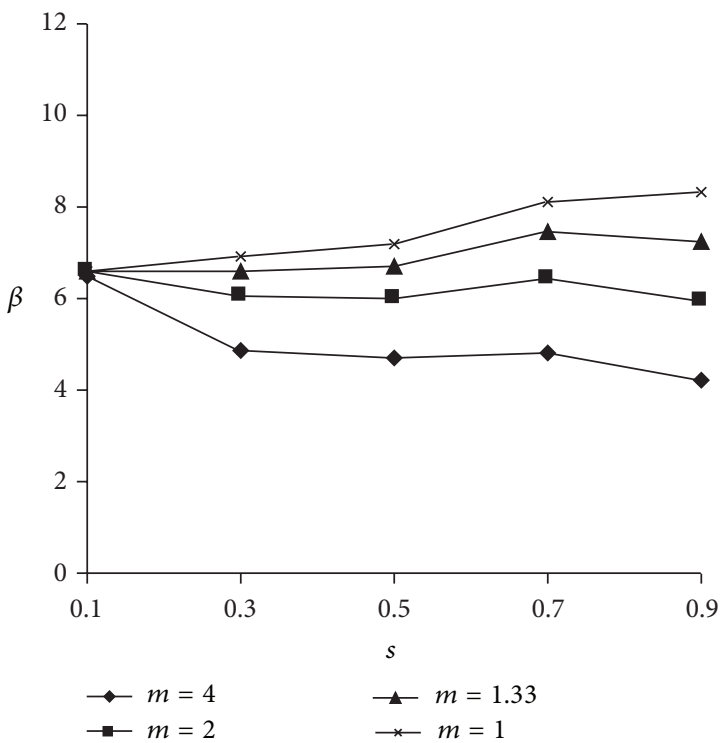

(c) $n=2$

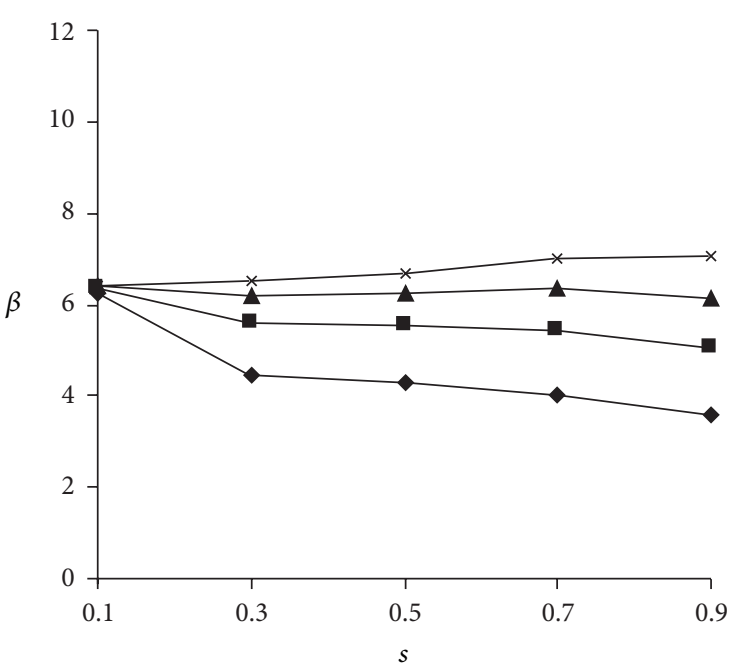

(b) $n=1.33$

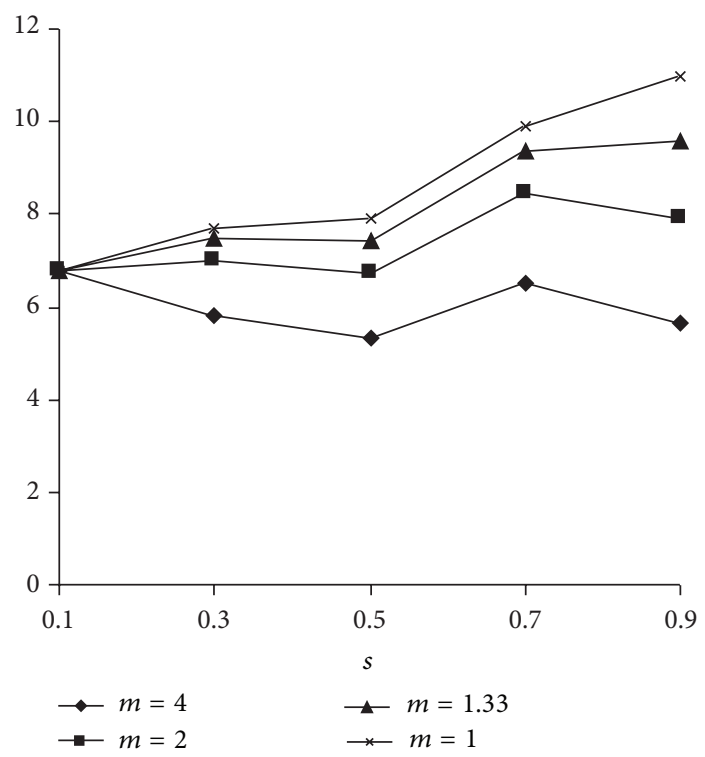

(d) $n=4$

FIGURE 4: VIM results for $\beta$ values for two-segment stepped columns with clamped-clamped (C-C) ends for different values of load ratio $(m)$, stiffness ratio $(n)$, and length ratio $(s)$.

conducted using VIM, and buckling loads for this special type of nonuniform columns are tabulated for various values of stiffness, load, and length ratios. The tabulated values can directly be used by design engineers who use such members in their structural/mechanical system. VIM results are also compared with the exact results available in the literature for some special cases to verify the efficiency of the technique to analyze this special stability problem.

\section{References}

[1] H. Saffari, R. Rahgozar, and R. Jahanshahi, "An efficient method for computation of effective length factor of columns in a steel gabled frame with tapered members," Journal of Constructional Steel Research, vol. 64, no. 4, pp. 400-406, 2008.

[2] T. V. Galambos, Guide To Stability Design Criteria For Metal Structures, John Wiley \& Sons, 5th edition, 1998.
[3] J. H. He, "Variational iteration method-a kind of non-linear analytical technique: some examples," International Journal of Non-Linear Mechanics, vol. 34, no. 4, pp. 699-708, 1999.

[4] S. B. Coşkun and M. T. Atay, "Determination of critical buckling load for elastic columns of constant and variable cross-sections using variational iteration method," Computers and Mathematics with Applications, vol. 58, no. 11-12, pp. 2260-2266, 2009.

[5] M. T. Atay and S. B. Coşkun, "Elastic stability of Euler columns with a continuous elastic restraint using variational iteration method," Computers and Mathematics with Applications, vol. 58, no. 11-12, pp. 2528-2534, 2009.

[6] F. Okay, M. T. Atay, and S. B. Coçkun, "Determination of buckling loads and mode shapes of a heavy vertical column under its own weight using the variational iteration method," International Journal of Nonlinear Sciences and Numerical Simulation, vol. 11, no. 10, pp. 851-857, 2010. 
[7] S. Pinarbasi, "Lateral torsional buckling of rectangular beams using variational iteration method," Scientific Research and Essays, vol. 6, no. 6, pp. 1445-1457, 2011.

[8] S. Pinarbasi, "Buckling analysis of nonuniform columns with elastic end restraints," Journal of Mechanics of Materials and Structures, vol. 7, no. 5, pp. 485-507, 2012.

[9] J. H. He, G. C. Wu, and F. Austin, "The variational iteration method which should be followed," Nonlinear Science Letters A, vol. 1, no. 1, pp. 1-30, 2010.

[10] S. P. Timoshenko and J. M. Gere, Theory of Elastic Stability, McGraw-Hill, New York, NY, USA, 2nd edition, 1961.

[11] C. M. Wang, C. Y. Wang, and J. N. Reddy, Exact Solutions For Buckling of Structural Members, CRC Press, Boca Raton, Fla, USA, 2005. 


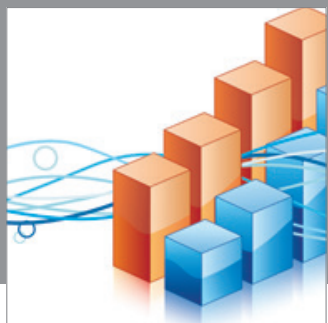

Advances in

Operations Research

mansans

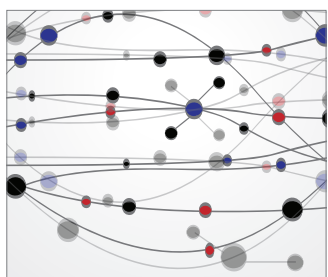

The Scientific World Journal
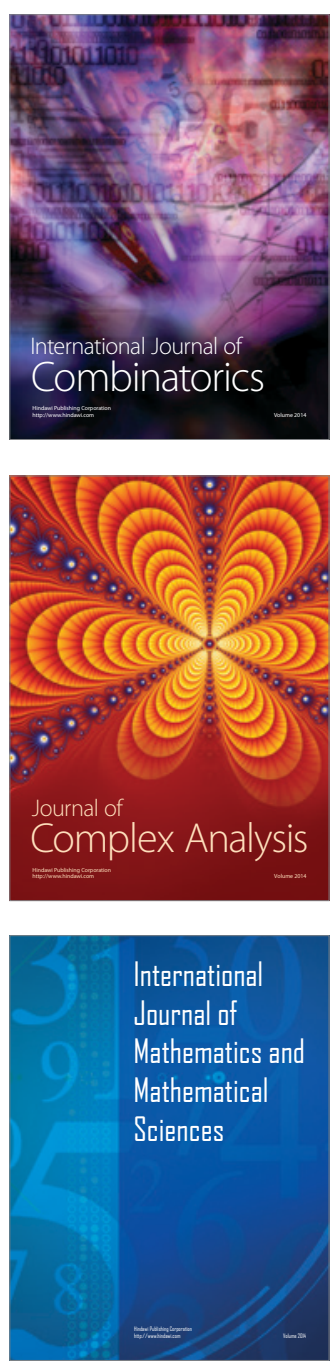
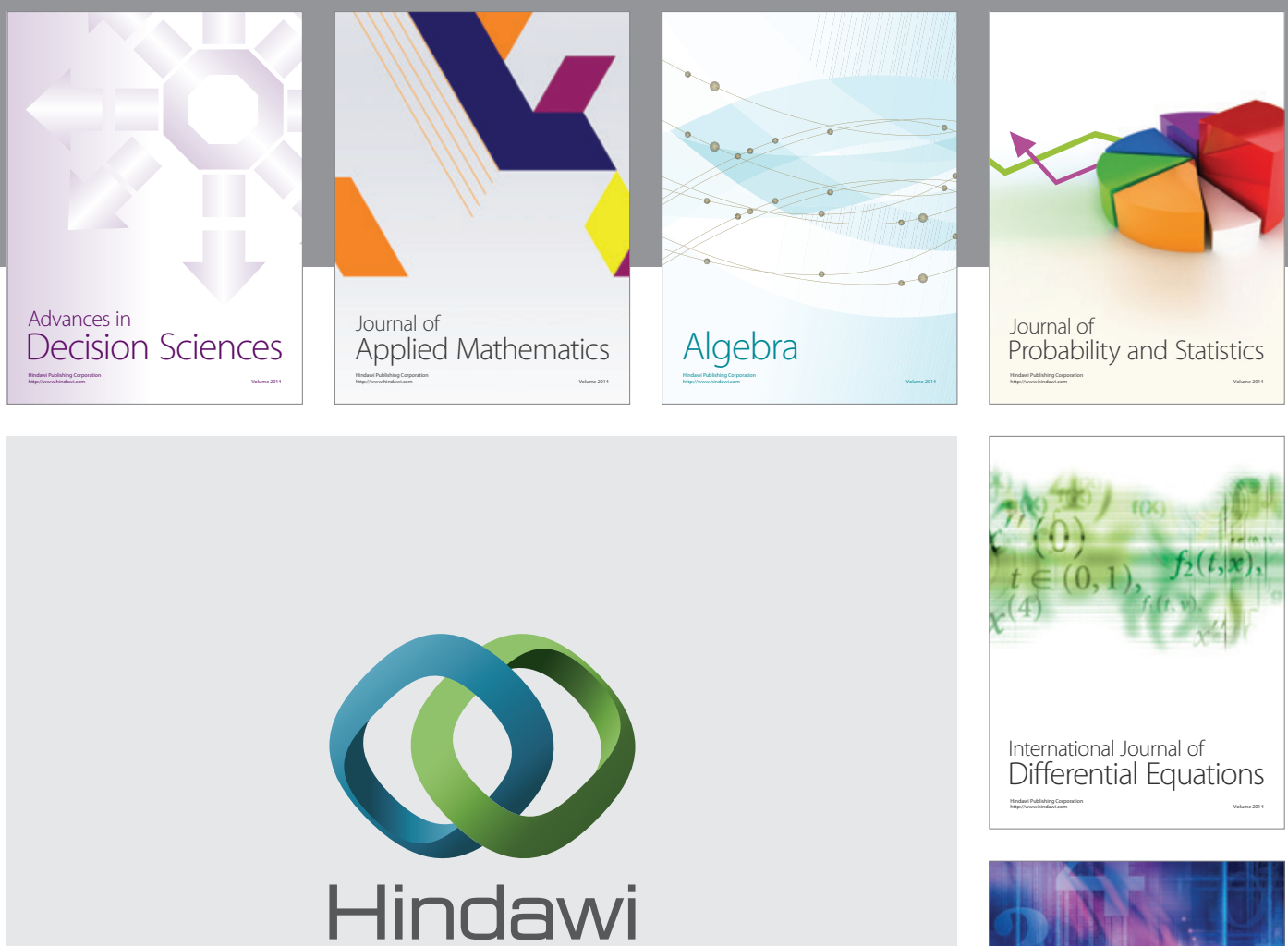

Submit your manuscripts at http://www.hindawi.com
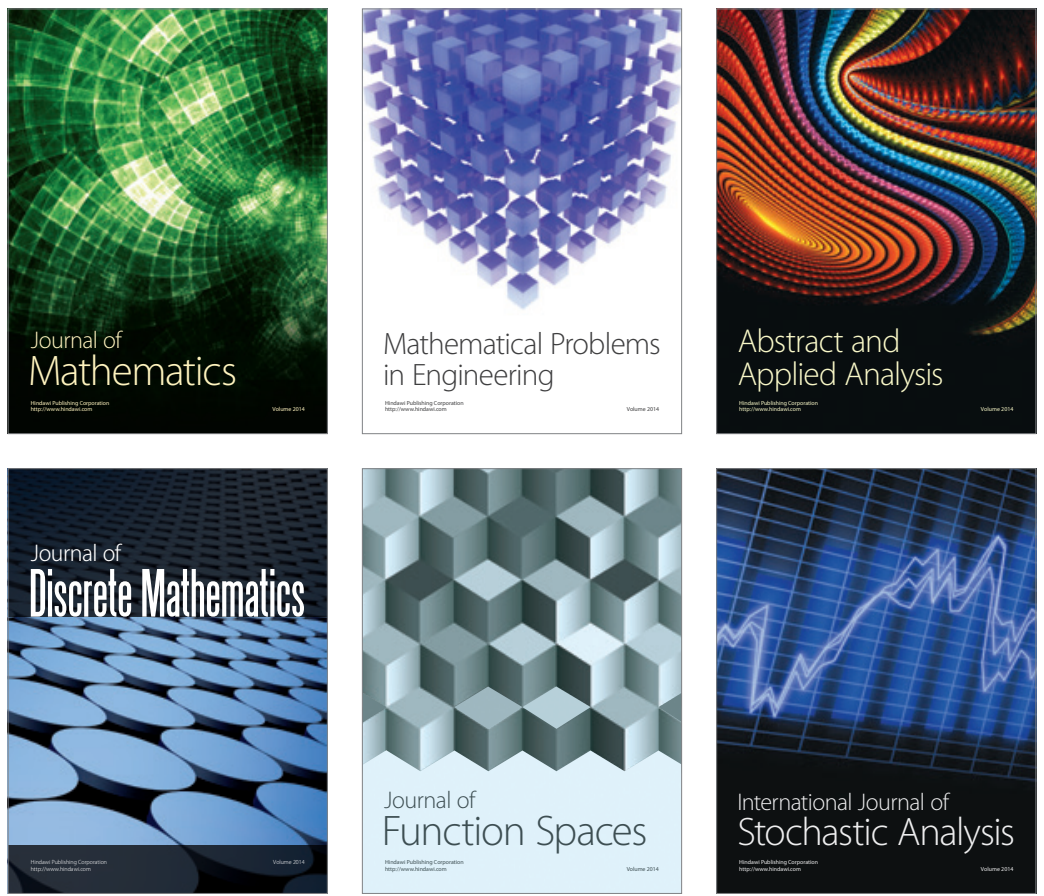

Journal of

Function Spaces

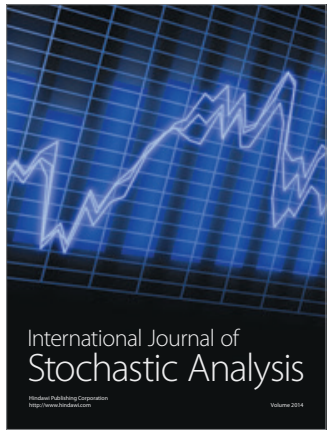

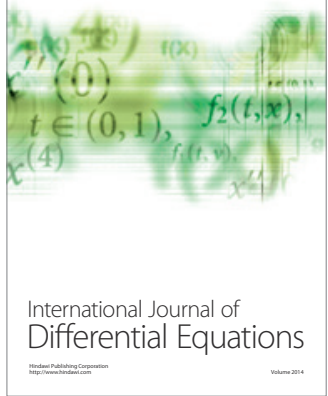
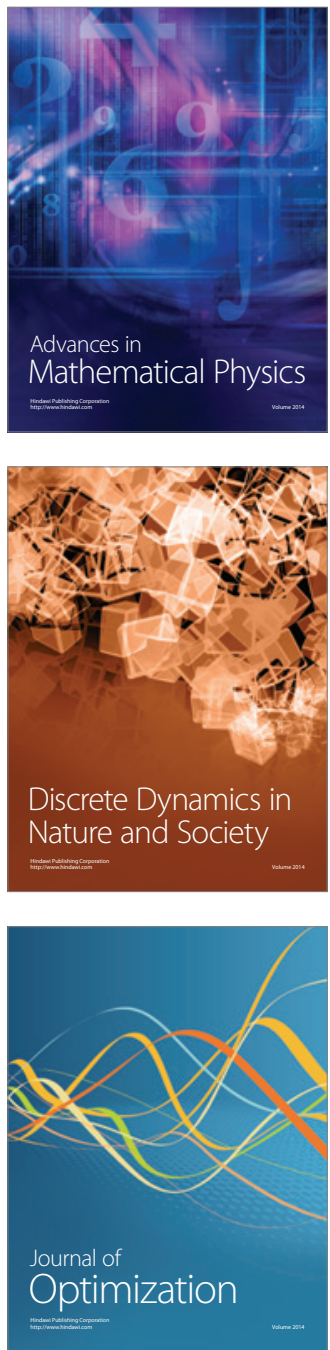